\title{
Producción de frutos de uchuva (Physalis peruviana L.) bajo diferentes láminas de riego, frecuencias de riego y dosis de calcio
}

\section{Production of cape gooseberry (Physalis peruviana L.) fruits with different irrigation coefficients and frequencies and doses of calcium}

JAVIER GIOVANNI ÁLVAREZ-HERRERA', 4

GERHARD FISCHER ${ }^{2}$

JAVIER ENRIQUE VÉLEZ-SANCHEZ ${ }^{3}$

Tratamiento de uchuva con baja lámina de agua y deficiencia de calcio.

Foto: J.G. Álvarez-Herrera

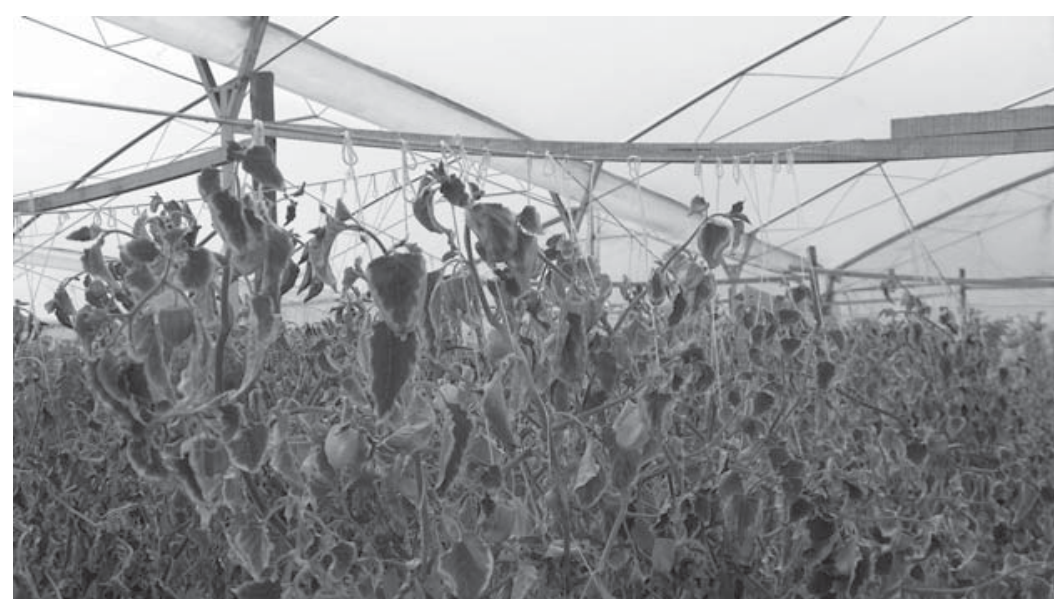

\section{RESUMEN}

La uchuva es la fruta exótica más importante para Colombia y constituye un renglón de exportaciones importante, no obstante en los últimos años el rendimiento obtenido en el cultivo ha disminuido. Por lo anterior, es necesario evaluar factores que afecten la producción, como lo son el riego y la nutrición con calcio, para lo cual se empleó un diseño en bloques al azar con 12 tratamientos en arreglo factorial de $4 \times 3$. Los bloques fueron las frecuencias de riego (4, 9 y 14 días distanciadas). El primer factor fue la lámina de riego $(0,7 ; 0,9 ; 1,1$ y 1,3 de la evaporación del tanque clase A) y el segundo la dosis de calcio $(0,50$ y $100 \mathrm{~kg} \mathrm{ha-1),} \mathrm{lo} \mathrm{que} \mathrm{representó} 36$ unidades experimentales. Se transplantó en materas de $20 \mathrm{~L}$ usando turba rubia como sustrato. El porcentaje de frutos obtenidos para tamaños grande $(>22 \mathrm{~mm})$, mediano $(18-22 \mathrm{~mm})$ y pequeño $(<18 \mathrm{~mm})$ fue de $9,17 \%$, $31,44 \%$ y $59,75 \%$, respectivamente. Con intervalos de riego más distantes, la cantidad de frutos pequeños incrementó, no obstante, si se aplicó $\mathrm{Ca}^{2+}$, el porcentaje de frutos pequeños disminuyó. La frecuencia de riego cada 4 días presentó los frutos con mayor masa fresca. Al aumentar la cantidad de agua aplicada con un coeficiente de riego de 1,3, creció el número de frutos y la producción por planta. La acumulación de masa seca en el fruto aumentó conforme creció el coeficiente de riego y con la frecuencia de riego más prolongada.

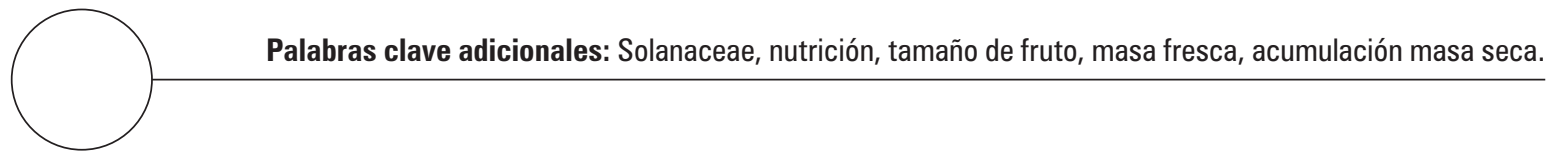

Facultad de Ciencias Agropecuarias, Grupo de Investigaciones Agrícolas, Universidad Pedagógica y Tecnológica de Colombia (UPTC), Tunja (Colombia).

2 Facultad de Ciencias Agrarias, Departamento de Agronomía, Universidad Nacional de Colombia, Bogotá (Colombia).

3 Facultad de Ingeniería, Departamento de Ingeniería Civil e Ingeniería Agrícola, Universidad Nacional de Colombia, Bogotá (Colombia).

4 Autor para correspondencia. jgalvarezh@gmail.com 


\section{ABSTRACT}

The cape gooseberry is the most important exotic fruit for Colombia and is a major export item; however, in recent years, the yield of this crop has decreased. Therefore, it is necessary to assess factors that affect performance, such as irrigation and calcium nutrition, for which a randomized block design with 12 treatments in a $4 \times 3$ factorial arrangement was used. The blocks were the irrigation frequencies (4, 9 and 14 day intervals). The first factor was the irrigation level (0.7, 0.9, 1.1, and 1.3 of Class A pan evaporation) and the second one was the dose of calcium $\left(0,50\right.$ and $\left.100 \mathrm{~kg} \mathrm{ha}^{-1}\right)$, representing 36 experimental units. The cape gooseberries were transplanted into $20 \mathrm{~L}$ pots using peat moss as the substrate. The percentage of fruits obtained for the large $(>22 \mathrm{~mm})$, medium $(18-22 \mathrm{~mm})$ and small $(<18 \mathrm{~mm})$ sizes was $9.17 \%, 31.44 \%$ and $59.75 \%$, respectively. As the irrigation frequency increased, the fruit production was higher; however, if $\mathrm{Ca}^{2+}$ was applied, the amount of small fruits decreased. The irrigation frequency of 4 days had the fruits with the highest average fresh mass. By increasing the amount of applied water with a 1.3 irrigation coefficient, the production per plant increased along with the number of fruits, especially small fruits. The accumulation of dry mass in the fruits increased with the irrigation level and longer irrigation frequencies.

Additional key words: Solanaceae, nutrition, fruit size, fresh weight, dry matter accumulation.

El mercado de frutas tropicales en estado fresco a nivel mundial ha mostrado un crecimiento acelerado en los últimos años, así mismo la demanda de frutas exóticas dentro de las cuales se encuentra la uchuva cuya exportación a los países desarrollados es cada vez mayor (Fischer et al., 2014). Aún más, la eliminación de los tratamientos cuarentenarios de la uchuva exportada de Colombia a Estados Unidos crea un mercado importante el cual hay que satisfacer. Según Agronet (2015), a pesar de que el área sembrada del cultivo de uchuva ha aumentado en el periodo comprendido entre 2010 y 2013, de 745 a 880 ha, el rendimiento se ha reducido de 16,1 a 14,6 $\mathrm{t} \mathrm{ha}^{-1}$, lo que constituye una considerable disminución del 9,3\%, probablemente ocasionada por un aumento en el rajado de los frutos y por otros aspectos como una adecuada fertilización y control de enfermedades.

Los frutos de uchuva son casi redondos y pesan entre 4 y $10 \mathrm{~g}$ en cultivos comerciales, necesitan entre 60 y 80 días para madurar (Ramírez et al.,
2013). El cáliz encierra el fruto totalmente hasta su madurez y pierde su clorofila a partir de unos 40-45 d de su desarrollo (Fischer et al., 2014), protegiéndolo contra insectos/plagas y condiciones climáticas adversas, además de ser un empaque único y ecológico en la poscosecha (Fischer et al., 1997).

En un primer ensayo con uchuva, a nivel de campo, láminas de riego del 1,2 (120\% de la evaporación), aumentaron el rendimiento de frutos por planta, en la parcela en la cual se adicionó $\mathrm{Ca}$, comparado con la aplicación de una menor cantidad de agua (Gordillo et al., 2004), no obstante, bajo invernadero y con una aplicación de un coeficiente de riego neto de 1,3 y $100 \mathrm{~kg} \mathrm{ha}^{-1}$ de Ca, la producción disminuyó así como el porcentaje de frutos rajados en uchuva (Álvarez et al., 2012).

Entre todos los recursos que exige una planta para su crecimiento, desarrollo y funciones, el agua es el de mayor cantidad, con el agravante 
que puede ser el más limitante, lo que acentúa la importancia del riego y su papel clave en la producción de los cultivos (Taiz y Zeiger, 2010).

Según Álvarez et al. (2012), los frutos de uchuva presentan problemas de rajado, para lo cual se recomienda aplicar en la fertilización calcio, y realizar un suministro de riego constante, no obstante no se conoce el efecto de estos factores en la producción de uchuva. Por lo anterior, el objetivo de este trabajo fue conocer el efecto de diferentes láminas de riego, frecuencias de riego y dosis de calcio sobre la producción de frutos de uchuva con el fin de tomar decisiones importantes sobre el manejo del cultivo con el fin de aumentar la producción.

\section{MATERIALES Y MÉTODOS}

El experimento se desarrolló en un invernadero de plástico de la Facultad de Ciencias Agrarias de la Universidad Nacional de Colombia, sede Bogotá, ubicado a una altitud de $2.556 \mathrm{msnm}$. La temperatura de invernadero promedio fue de $18^{\circ} \mathrm{C}$ y la humedad relativa de un $60 \%$.

Se utilizó un diseño experimental en bloques completos al azar, con 12 tratamientos. El criterio de bloqueo fueron las frecuencias de riego (4, 9 y $14 \mathrm{~d}$ ). Los tratamientos estuvieron constituidos por un arreglo factorial de $4 \times 3$, en donde el primer factor correspondió a las láminas de riego aplicadas (coeficientes netos 0,7; 0,9; 1,1 y 1,3 de la evaporación del tanque evaporímetro clase A) $\mathrm{y}$ el segundo factor a las dosis de calcio $(0,50 \mathrm{y}$ $100 \mathrm{~kg} \mathrm{ha}^{-1}$ ), cada bloque supuso una repetición, lo que generó 36 unidades experimentales (UE). Cada UE estuvo compuesta por dos plantas de uchuva, para un total de 72 plantas, las cuales se sembraron en materas de $80 \mathrm{~cm}$ de diámetro y $50 \mathrm{~cm}$ de profundidad. Se utilizó turba rubia como sustrato.

El material vegetal sembrado fue la especie Physalis peruviana L., ecotipo Colombia. Las plantas, propagadas por semilla, fueron transplantadas en materas de $20 \mathrm{~L}$ de capacidad a una distancia de $2 \mathrm{~m}$ entre plantas y $2 \mathrm{~m}$ entre surcos, y se realizaron las labores culturales tradicionales (manejo fitosanitario, podas y cosecha) de los cultivos comerciales de las zonas productoras. La fertilización se realizó aplicando las siguientes dosis en $\mathrm{kg} \mathrm{ha}^{-1}$ : $150 \mathrm{~N}, 220 \mathrm{P}_{2} \mathrm{O}_{5}, 150 \mathrm{~K}_{2} \mathrm{O}, 60$ $\mathrm{MgO}, 40 \mathrm{~S}, 1 \mathrm{~B}, 3 \mathrm{Zn}, 2 \mathrm{Cu}$, y $0,5 \mathrm{Mn}$, fraccionada desde el momento de la siembra, primero en dos aplicaciones trimestrales y luego tres aplicaciones bimensuales. Se utilizó el sistema de tutorado en $\mathrm{V}$ alto (Angulo, 2005).

Para la aplicación de las láminas de riego se empleó un sistema de riego por goteo (dos goteros por planta con un caudal de $4 \mathrm{~L} \mathrm{~h}^{-1}$ ). Una vez sembradas las plantas, se aplicaron las diferentes dosis de calcio establecidas en corona incorporándolas al sustrato, repartidas mensualmente debido a la baja movilidad que presenta este elemento en el suelo. Se midió cada $4 \mathrm{~d}$ la evaporación en un tanque evaporímetro tipo A construido a escala 1:1 e instalado dentro del invernadero con el fin de establecer la cantidad de agua a aplicar, según la ecuación de cálculo de uso consuntivo que tiene en cuenta la evapotranspiración potencial.

Las aplicaciones se realizaron con base en la siguiente formula:

Lámina $=\frac{\operatorname{Etp} * \mathrm{C} * \mathrm{~A}}{\eta_{\mathrm{r}}}$

En donde, Etp = evapotranspiración en $\mathrm{mm}$ medida en el tanque evaporímetro; $C=$ coeficiente multiplicador según tratamientos; $A=$ área de la matera $\left(254,4 \mathrm{~cm}^{2}\right) ; \eta_{r}=$ eficiencia del riego por goteo $(0,9)$.

La medición de las diferentes variables de respuesta se realizó con los frutos obtenidos de ocho cosechas efectuadas en el cultivo quincenalmente, usando como índice de madurez el color del cáliz (Icontec, 1999). La primera cosecha se realizó a la semana 17 después de transplante (ddt). 
Se midió la producción por calidades comerciales $(\mathrm{kg})$, esta se determinó mediante el porcentaje de la masa fresca de los frutos por planta de cada una de las categorías según norma Icontec NTC 4580 (Icontec, 1999). Los frutos fueron clasificados en tres tamaños según el diámetro equatorial: $>22 \mathrm{~mm}$, grandes; $18-22 \mathrm{~mm}$, medianos; $<18 \mathrm{~mm}$, pequeños.

La masa fresca promedio de frutos se determinó pesando el total de frutos (sin cáliz) por planta y dividiéndolo por el número de frutos para cada tratamiento en una balanza electrónica Acculab VIC 612 de 0,01g de precisión (Sartorius Spain, Madrid). La producción de frutos por planta se calculó pesando el número total de frutos sin cáliz por planta. El porcentaje de masa seca del fruto se calculó dividiendo la masa seca de frutos en la masa fresca del fruto y se expresó en porcentaje. La masa seca de frutos se obtuvo luego de secar los frutos a $70^{\circ}$ durante $48 \mathrm{~h}$ y posteriormente determinarles la masa.

Luego de la prueba de Kolmogorv-Smirnov realizada con el fin de comprobar los supuestos de normalidad, se hizo un análisis de varianza (Anova) para un diseño en bloques completamente al azar con el fin de determinar si hubo diferencias significativas entre frecuencias de riego (bloques) y entre tratamientos (lámina por frecuencia) para cada una de las variables de respuesta medidas, adicionalmente se hizo una prueba de comparación de promedios de Tukey al 5\%, para clasificar cada uno de los niveles de los factores evaluados. El análisis de los datos se realizó con el programa SAS v. 9.2.

\section{RESULTADOS Y DISCUSIÓN}

\section{Producción por calidades comerciales}

El porcentaje de frutos de tamaño grande no presentó diferencias estadísticas significativas en el Anova de tratamientos, ni para cada uno de los factores evaluados. El porcentaje de frutos grandes obtenido para todas las cosechas fue de 9,17\%. El porcentaje de frutos recolectados de tamaño mediano (18-22 mm) fue del 31,44\%, y tampoco presentó diferencias significativas; no obstante, al analizar el factor calcio por separado, este afectó significativamente el porcentaje de frutos medianos, ya que al no aplicar calcio se obtuvo $26 \%$ de frutos medianos, mientras que al aplicar 50 y $100 \mathrm{~kg} \mathrm{ha}^{-1}$ de $\mathrm{Ca}^{2+}$ al suelo, el porcentaje de frutos medianos aumentó un 36\% y $32 \%$, respectivamente. El porcentaje de frutos clasificados como pequeños $(<18 \mathrm{~mm})$ presentó diferencias significativas para la frecuencia de riego como las dosis de $\mathrm{Ca}^{2+}$. El porcentaje de frutos pequeños obtenidos fue del $59,75 \%$.

En la tabla 1 se puede apreciar que a medida que la frecuencia de riego aumenta (intervalos de riego más cortos), la cantidad de frutos pequeños se incrementa significativamente. Este resultado también se ve reflejado por la tendencia de que los frutos de tamaños medianos y grandes disminuyeron a intervalos de riego más amplios. Esto implica que la producción de frutos de uchuva se ve afectada principalmente por el tamaño de estos, resultado similar a lo expuesto por Léchaudel y Joas (2007), quienes mencionan que en mango, al aumentar la frecuencia de riego el tamaño de los frutos aumenta. Esta diferencia en el tamaño de los frutos ocurre, probablemente, porque al disminuir la frecuencia de riego (intervalos de riego más largos), la planta experimenta un estrés hídrico que ralentiza la asimilación de carbono y la entrada de agua hacia el fruto, debido a la disminución en la conductancia estomática y en el potencial hídrico de las hojas, el cual es ocasionado porque las raíces al estar bajo condición de déficit de agua producen ABA (Balaguera et al., 2008), el cual genera una señal que reduce la apertura estomática, la cual disminuye la fotosíntesis (Ramachandra et al., 2004), así como también la elongación celular y la asimilación de sustratos.

En la tabla 1 también se puede apreciar que a menor dosis de calcio aplicado mayor fue el por- 


\section{Tabla 1. Efecto del coeficiente de riego, la dosis de calcio y la frecuencia de riego en el porcentaje de frutos de uchuva, clasificados por tamaño, de plantas sembradas en turba rubia bajo invernadero.}

\begin{tabular}{|c|c|c|c|c|}
\hline \multirow[b]{2}{*}{ Factor } & \multirow{2}{*}{$\begin{array}{c}\text { Nivel } \\
\text { del } \\
\text { factor }\end{array}$} & \multicolumn{3}{|c|}{ Tamaño del fruto } \\
\hline & & $\begin{array}{c}\text { Grande } \\
(>22 \mathrm{~mm})\end{array}$ & $\begin{array}{c}\text { Mediano } \\
(18-22 \mathrm{~mm})\end{array}$ & $\begin{array}{l}\text { Pequeño } \\
(<18 \mathrm{~mm})\end{array}$ \\
\hline \multirow{4}{*}{ Coeficiente de riego } & 0,7 & 8,128 a & 27,521 a & 64,438 a \\
\hline & 0,9 & 9,503 a & 32,425 a & 57,918 a \\
\hline & 1,1 & 9,982 а & 30,645 a & 59,762 a \\
\hline & 1,3 & 9,086 a & 34,880 a & 56,884 a \\
\hline \multirow{3}{*}{ Dosis de calcio $\left(\mathrm{kg} \mathrm{ha}^{-1}\right)$} & 0 & 10,747 a & $26,027 \quad b$ & 63,604 a \\
\hline & 50 & 8,314 a & 36,186 a & $55,764 \quad b$ \\
\hline & 100 & 8,463 a & 32,116 ab & $59,883 \mathrm{ab}$ \\
\hline \multirow{3}{*}{ Frecuencia de riego (días) } & 4 & 12,113 a & 32,826 a & 56,053 b \\
\hline & 9 & 8,113 a & 33,232 a & $58,608 \mathrm{ab}$ \\
\hline & 14 & 7,298 a & 28,270 a & 64,590 a \\
\hline
\end{tabular}

Promedios con letras distintas, en la misma columna y clasificados por factor, indican diferencia significativa según la prueba de Tukey $(P \leq 0,05)$.

centaje de frutos pequeños, lo que implica que la aplicación de $\mathrm{Ca}^{+}$tuvo un efecto directo sobre la producción de frutos de tamaño grande y mediano. Estos resultados son similares a los encontrados en uchuva por Cooman et al. (2005), donde la ausencia de $\mathrm{Ca}^{2+}$ en la solución nutritiva causó frutos de menor tamaño, resultado que confirmaron Ciccarese et al. (2013) en frutos de uva, mientras Martínez et al. (2008) observaron un menor rendimiento en uchuva, debido a un reducido número de frutos, cuando se regaron las plantas sin $\mathrm{Ca}^{2+}$ en la solución. Del mismo modo, Román y Gutiérrez (1998), al aplicar $\mathrm{Ca}^{2+}$ en melón, encontraron que el tamaño de los frutos también aumentó. Esto se puede explicar basado en que una deficiencia de $\mathrm{Ca}^{2+}$ restringe la división celular y causa pérdida de integridad de la pared celular y el plasmalema (Dris, 2001), así mismo, si el $\mathrm{Ca}^{2+}$ se encuentra disponible en los estados iniciales, mayor cantidad de tejido en las paredes celulares se formará (Ciccarese et al., 2013), lo que implica que aplicar $\mathrm{Ca}^{2+}$ favorece el aumento en el tamaño de los frutos o al menos disminuye la probabilidad de que se presente un gran número de frutos pequeños.

\section{Masa fresca del fruto}

Esta variable presentó diferencias estadísticas altamente significativas $(P \leq 0,0028)$, sin embargo, el efecto del factor frecuencia de riego, al ser analizado por separado, fue el único que mostró un efecto significativo. La masa fresca promedio de los frutos fue de 3,55 $\pm 0,14 \mathrm{~g}$, valores similares a los reportados por Villamizar (1993) de 3,29 g y Cooman et al. (2005) de $4 \mathrm{~g}$ que son producciones menores que normalmente se cosechan en campo abierto (Gordillo et al., 2004). En la figura 1 se puede observar que a medida que disminuye la frecuencia de riego, por un riego más distante, la masa promedio de frutos fue menor, similar a lo reportado en mango por Spreer et al. (2007), quienes mencionan que al aplicar el riego más frecuentemente, aumentó la producción y el tamaño de los frutos.

También, este resultado concuerda con lo encontrado por Guerrero et al. (2007) en una finca en Machetá (Cundinamarca) con un suelo que presentaba entre 10 y $25 \%$ de humedad (frecuencias de riego más largas), y en donde el porcentaje de 


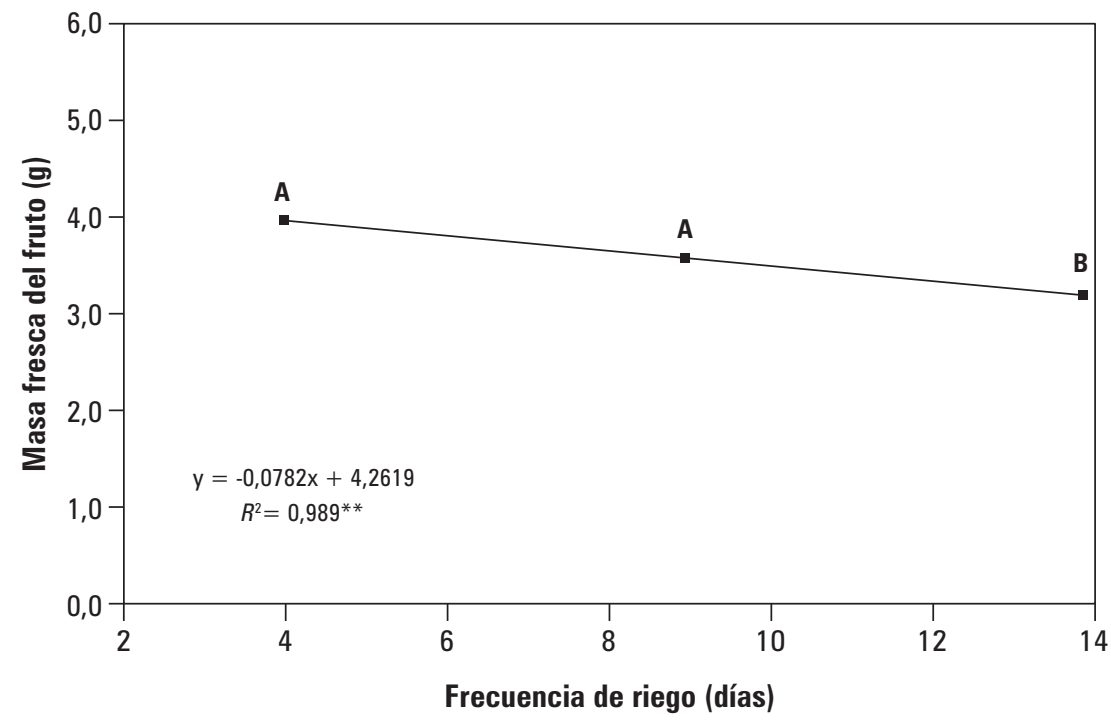

Figura 1. Efecto de la frecuencia de riego en la masa fresca de frutos en plantas de uchuva, sembradas en turba rubia bajo invernadero. Promedios con letras distintas indican diferencia significativa según la prueba de Tukey $(P \leq 0,05)$.

frutos de uchuva grandes y de exportación disminuyó considerablemente comparado con suelos de humedades más altas.

Del mismo modo, es probable que la aplicación del riego (con la solución nutritiva) a mayor frecuencia favoreció una concentración de nutrientes más estable en el sustrato, con lo cual la disponibilidad de estos para las plantas fue mayor, y tanto los procesos de asimilación como de traslocación de asimilados hacia los frutos se realizaron de forma más eficiente (Silber y Bar-Tal, 2008) y, en consecuencia, aumentó la masa de los frutos. Taiz y Zeiger (2010) aclaran que una suficiente cantidad de agua es necesaria, entre otras funciones, para el desarrollo de la turgencia que es la base para muchos procesos fisiológicos, incluyendo el alargamiento celular y el transporte de los carbohidratos en el floema para el llenado del fruto.

Las plantas de uchuva al tener un suministro irregular de agua debido a las bajas frecuencias de riego a menudo sufrieron de estrés hídrico, esto probablemente generó una menor área foliar y un cierre estomático más pronunciado y frecuente (Fischer y Orduz-Rodríguez, 2012). Epstein y Bloom (2005) mencionan que cuando existe una señal de estrés a nivel radicular, el pH del flujo xilemático aumenta, lo que permite una acumulación de ABA en el apoplasto, el cual genera una señal que es captada por los receptores de las células guarda. Esto hace que la membrana se vuelva más permeable al potasio, con lo cual ocurre el cierre estomático y, en consecuencia, la disminución del área foliar, esto disminuye la transferencia de azúcares de los órganos fuente hacia los frutos, lo que finalmente, se ve reflejado en un menor tamaño y acumulación de masa fresca por parte de los frutos (Zegbe-Domiguze et al., 2004). Del mismo modo, Álvarez-Herrera et al. (2012) encontraron en uchuva que el porcentaje de frutos de tamaño pequeño, recolectado a lo largo de todo el experimento fue de casi un $60 \%$, y al observar el peso fresco de frutos, los frutos obtenidos de las plantas regadas cada $14 \mathrm{~d}$ presentaron en promedio 3,14 g. 
Así mismo, Acevedo-Opazo et al. (2010) aplicaron diferentes regímenes de agua en uva y encontraron que con la menor aplicación de agua, el tamaño y la masa de los frutos se vio disminuido en un 6\%. De igual modo, Fallahi et al. (2010) mencionan que al aplicar menor cantidad de agua la producción de manzana disminuyó, reduciendo el tamaño de los frutos, mientras las hojas presentaron senescencia prematura; no obstante, el color de los frutos fue más intenso, posiblemente por un efecto de concentración, como lo observaron Fischer y Martínez (1999) en uchuvas maduras y encogidas.

\section{Producción de frutos}

Esta variable presentó diferencias altamente significativas $(P \leq 0,0035)$. Al evaluar por separado los factores, la lámina de riego y la frecuencia de riego afectaron la producción total/planta de forma significativa, mientras que la dosis de calcio no afectó la variable producción. En la figura 2A se observa que a medida que se incrementa la lámina de riego, la producción de uchuva aumenta, al pasar de $486 \mathrm{~g}$ para el coeficiente de 0,7 a 1.011,8 g por planta para el coeficiente de 1,3. Estos da- tos de producción son bajos si se comparan con los obtenidos por Angulo (2005), quien encontró valores de $900 \mathrm{~g}$ por planta para aquellas a libre exposición, mientras Martínez et al. (2008) reportan valores que van desde los $1.884 \mathrm{~g}$ hasta los 2.649 g por planta, teniendo en cuenta la duración y las condiciones de estos estudios experimentales.

Nuestros resultados son similares a los registrados por Gordillo et al. (2004) en uchuva, creciendo en campo abierto, y el mismo comportamiento reportan Treeby et al. (2007) en cítricos, Rufat et al. (2010) en durazno y Chun-Zhi et al. (2009) en melón, entre otros cultivos, quienes mencionan que al aumentar la cantidad de agua aplicada se aumenta la producción.

Según Vélez y Álvarez (2012), una planta con un suministro hídrico adecuado posee a nivel celular un balance normal del agua que genera presión de turgencia, la cual es esencial para la realización de procesos fisiológicos como el alargamiento celular, intercambio gaseoso en las hojas, transporte en el floema, traslocación y distribución de fotoasimilados, así como la facilidad en el transporte a través de las membranas. Del
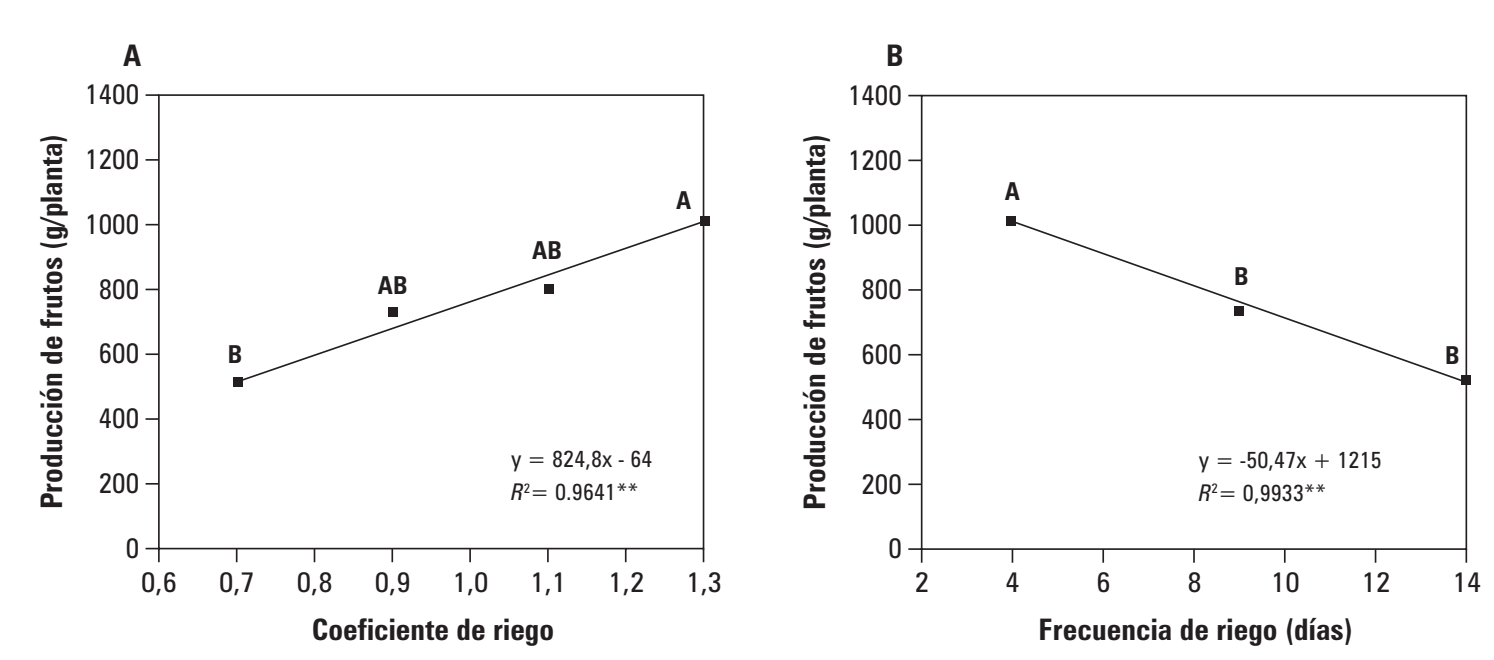

Figura 2. Efecto de A) la lámina de riego y B) la frecuencia de riego en la producción de frutos en plantas de uchuva, sembradas en turba rubia bajo invernadero. Promedios con letras distintas indican diferencia significativa según la prueba de Tukey $(P \leq 0,05)$. 
mismo modo, como mencionan estos autores, la presión de turgencia también contribuye a mantener la rigidez y la estabilidad mecánica de los tejidos y a garantizar un proceso eficiente de la fotosíntesis, lo cual redunda en un crecimiento óptimo de la planta y en la generación de estructuras reproductivas (flores y frutos) de mayor tamaño, que afectan positivamente la producción.

A medida que la frecuencia de riego se hizo más prolongada, la producción disminuyó en un $50 \%$ aproximadamente (figura 2B). Cuando se regó cada 4 d, la producción alcanzó 1.025 g por planta, mientras que cuando la frecuencia de riego fue de $14 \mathrm{~d}$, la producción fue de tan solo $520 \mathrm{~g}$. Lo anterior es similar a lo encontrado por Wang et al. (2006), quienes, al aumentar la frecuencia de riego en papa, obtuvieron mayor producción.

Del mismo modo, Léchaudel y Joas (2007) encontraron que al incrementar las aplicaciones de agua en el tiempo se aumentó la producción en mango, así como en marañon (Oliveira et al., 2006).

Cuando se manejan altas frecuencias de riego, sin afectar la concentración de oxígeno en la rizósfera, los patrones de humedad y la distribución del agua son uniformes, lo que conlleva a una adecuada distribución y crecimiento de raíces, esto favorece la disponibilidad y el transporte de nutrientes e influye en una mayor eficiencia del sistema radicular, densidad de pelos radiculares, y arquitectura de la raíces (Silber et al., 2003), situación que estimula la producción de la planta, y que pudo haber jugado un papel determinante en la rendimiento de la uchuva.

\section{Número de frutos por planta}

La lámina de riego y la frecuencia de riego mostraron un efecto altamente significativo $(P \leq 0,0013 ; P \leq 0,0005$, respectivamente) sobre el número de frutos por planta, sin embargo, la dosis de $\mathrm{Ca}^{2+}$ no tuvo ningún efecto marcado. El número de frutos cosechados por planta varió de 116 para una lámina de riego de 0,7 a 259 para una de 1,3 (figura $3 \mathrm{~A}$ ). Los resultados hallados son menores a los encontrados por Martínez et al. (2008), quienes probaron en uchuva tratamientos con una adecuada nutrición y obtuvieron 349 frutos por planta, mientras que cuando
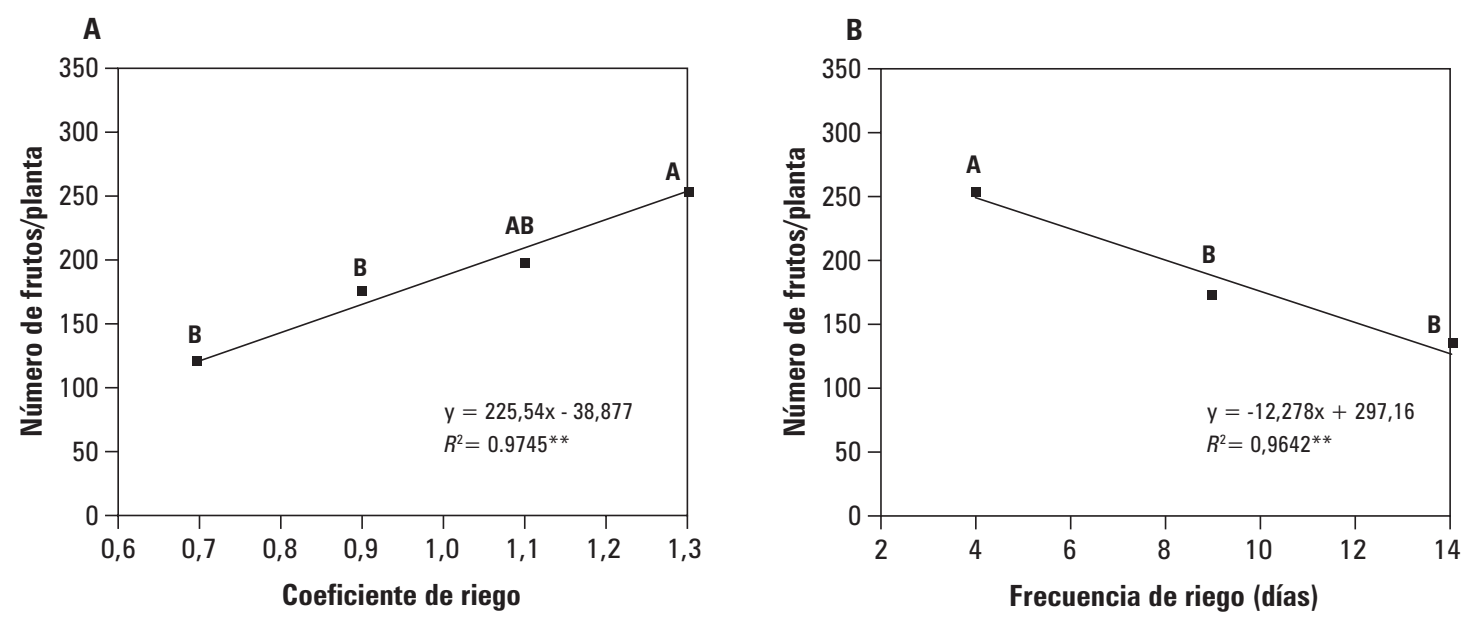

Figura 3. Efecto de A) la lámina de riego y B) la frecuencia de riego en el número de frutos en plantas de uchuva, sembradas en turba rubia bajo invernadero. Promedios con letras distintas indican diferencia significativa según la prueba de Tukey $(P \leq 0,05)$. 
las plantas tenían deficiencia cálcica, el número de frutos se redujo en un 25\%. A su vez, Fischer et al. (2007) encontraron en campo abierto en Boyacá, en un cultivo de 12 meses, que el ecotipo Colombia produjo en promedio 380 frutos por planta y es el ecotipo que mayor número de frutos desarrolló en comparación con los ecotipos Kenia y Sudáfrica.

Es probable que la menor lámina de riego haya generado un reducido crecimiento longitudinal de las ramas reproductoras, y teniendo en cuenta que la uchuva forma en cada nudo de una rama reproductiva un fruto (Ramírez et al., 2013), el número de frutos por planta se viera afectado al aplicar una menor cantidad de agua. Por el contrario, cuando el suministro de riego fue mayor (coeficiente de 1,3), las plantas presentaron mayor vigor y crecimiento de ramas longitudinales, por lo tanto más estructuras, que aumentaron el número de frutos cosechados.

De acuerdo con la figura 3B, cuando se aplicó agua cada $4 \mathrm{~d}$ el número de frutos fue de $254 \mathrm{y}$ disminuyó a 132 cuando el suministro hídrico fue aplicado cada $14 \mathrm{~d}$, sin contar, con la gran cantidad de pérdida de frutos por abscisión temprana en este tratamiento. Resultados similares observaron Léchaudel y Joas (2007) en mango, quienes afirman que al regar cada $7 \mathrm{~d}$, la producción y el número de frutos fue mayor que cuando se regó cada 14 d, también mencionan que la caída de frutos en este último tratamiento fue mayor, esto probablemente ocurrió porque se sintetiza una mayor cantidad de ácido abscísico (ABA) y etileno, producto del estrés al que es sometida la planta, lo cual induce la abscisión de los frutos en los estados iniciales de crecimiento (Eo y Lee, 2009).

\section{Acumulación de masa seca en el fruto}

La acumulación de masa seca (MS) en el fruto presentó diferencias significativas tanto para la lámina de riego (figura 4A) como para la frecuencia de riego (figura 4B). Conforme se aumentó la cantidad de agua aplicada, mayor fue el porcentaje de MS de los frutos (figura 4A). No obstante, en otros ensayos no aumentó el porcentaje de MS, como por ejemplo en la uva (Porro et al., 2010) y en tomate (Veit-Köhler et al., 1999), mientras que en este estudio cuando

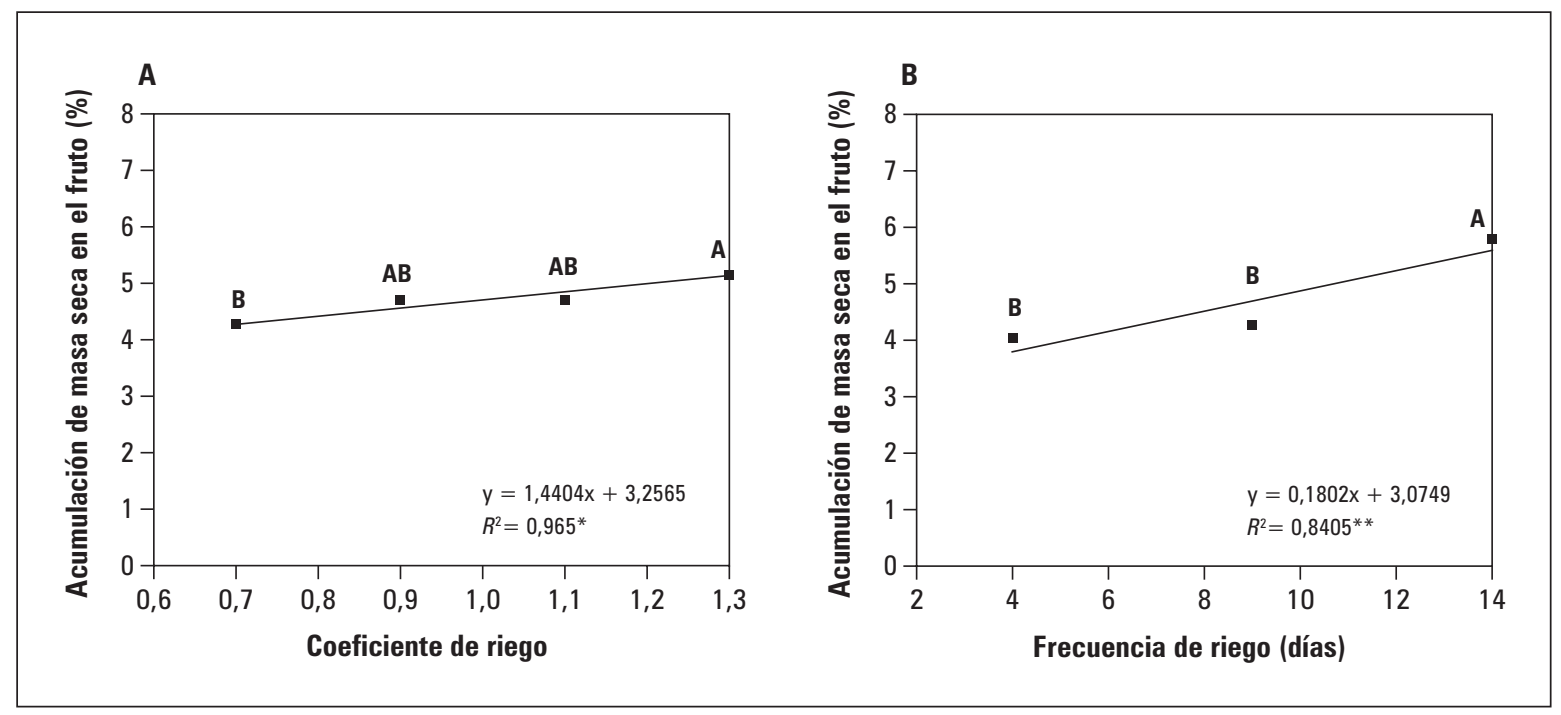

Figura 4. Efecto de A) la lámina de riego y B) la frecuencia de riego en la acumulación de masa seca en frutos de uchuva provenientes de plantas sembradas en turba rubia bajo invernadero. Promedios con letras distintas indican diferencia significativa según la prueba de Tukey $(P \leq 0,05)$. 
se incrementó la cantidad de agua aplicada, se considera que en la uchuva una mayor acumulación significativa de masa seca $(P \leq 0,05)$ en el fruto se dio debido a la aplicación del coeficiente de 1,3 (5,15\%), comparado con el de 0,9 (4,23\%), lo que puede ser el resultado de un estímulo del crecimiento foliar y consecuentemente de la cantidad de fotoasimilados producidos, traslocados y acumulados en el fruto, dentro de los cuales predomina la sacarosa (Fischer et al., 2015). También Fischer et al. (2007) atribuyen una mayor acumulación de MS en frutos de uchuva, en un sitio agroecológicamente más favorable, a una translocación más eficiente de fotoasimilados $y$, además, a una mayor fuerza de vertedero en condiciones óptimas.

El aumento significativo de la MS en los frutos, al aplicar frecuencias de riego de $14 \mathrm{~d}$ (figura 4B), puede ser un efecto de concentración, teniendo en cuenta que estos frutos desarrollaron la menor masa fresca en este tratamiento (figura 1) y, en consecuencia, acumularon la menor cantidad de agua y mayor MS.

\section{CONCLUSIONES}

- El incremento del coeficiente de riego y la frecuencia del riego (regar cada 4 y 9 d) aumentan el número de frutos por planta, pero solamente riegos más frecuentes aumentan la masa fresca de los mismos.

- El rendimiento por planta aumenta con un creciente coeficiente de riego, pero disminuye cuando se aplica el agua en intervalos más distantes.

- La acumulación de masa seca en el fruto crece con el coeficiente de riego, pero también con frecuencias de riego más distantes, este último resultado, posiblemente, por un efecto de concentración en los frutos más pequeños.

- Con un aumento de la dosis de calcio se disminuye el porcentaje de frutos pequeños y aumenta el número de frutos de tamaño mediano.

- Por último, en uchuva la aplicación de riego se justifica por el aumento de la producción.

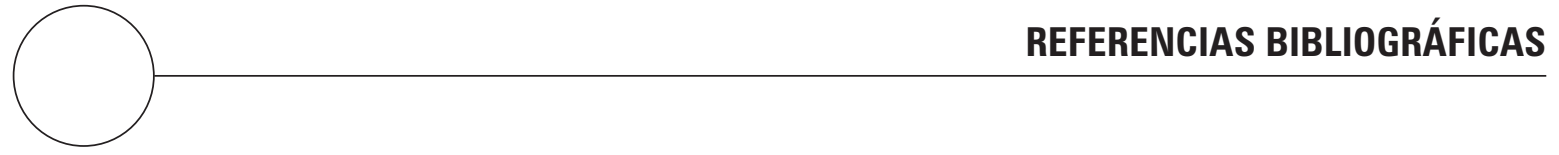

Acevedo-Opazo, C., S. Ortega-Farias y S. Fuentes. 2010. Effects of grapevine (Vitis vinifera L.) water status on water consumption, vegetative growth and grape quality: An irrigation scheduling application to achieve regulated deficit irrigation. Agr. Water Manage. 97, 956-964. Doi: 10.1016/j. agwat.2010.01.025

Agronet. 2015. Sistema de estadísticas agropecuarias. Producción nacional por producto. Uchuva. En: WwW.agronet.gov.co; consulta: agosto de 2015.

Álvarez-Herrera, J., H. Balaguera-López y G. Fischer. 2012. Effect of irrigation and nutrition with calcium on fruit cracking of the cape gooseberry (Physalis peruviana L.) in the three strata of the plant. Acta Hortic. 928, 163-170. Doi: 10.17660/ ActaHortic.2012.928.19
Angulo, R. 2005. Crecimiento, desarrollo y producción de la uchuva en condiciones de invernadero y campo abierto. pp. 111-129. En: Fischer, G., D. Miranda, W. Piedrahita y J. Romero (eds.). Avances en cultivo, poscosecha y exportación de la uchuva (Physalis peruviana L.) en Colombia. Unibiblos, Bogotá.

Balaguera, H.E., J.G. Álvarez-Herrera y J.D. Rodríguez. 2008. Efecto del déficit de agua en el trasplante de plántulas de tomate (Solanum lycopersicum L.). Agron. Colomb. 26(2), 246-255.

Ciccarese, A., A.M. Stellacci, G. Gentilesco y P. Rubino. 2013. Effectiveness of pre- and post-veraison calcium applications to control decay and maintain table grape fruit quality during storage. Postharvest Biol. Technol. 75, 135-141. Doi: 10.1016/j.postharvbio.2012.08.010 
Cooman, A., C. Torres y G. Fischer. 2005. Determinación de las causas del rajado del fruto de uchuva (Physalis peruviana L.) bajo cubierta. II. Efecto de la oferta de calcio, boro y cobre. Agron. Colomb. 23(1), 74-82.

Chun-Zhi, Z., B. Zhi-Long y Y. Bao-Zhong. 2009. Determination of optimum irrigation water amount for drip-irrigated muskmelon (Cucumis melo L.) in plastic greenhouse. Agr. Water Manage. 96, 595602. Doi: 10.1016/j.agwat.2008.09.019

Dris, R. 2001. Influence of calcium nutrition on the quality and postharvest behavior of apples. pp. 175-186. En: Dris, R., R. Niskanen y S. Jain (eds.). Crop management and postharvest handling of horticultural products. Vol. 1. Quality management. Science Publishers, Enfield, NH.

Epstein, E. y A. Bloom. 2005. Mineral nutrition of plants: principles and perspectives. $2^{\text {nd }} e d$. Sinauer Associates, Sunderland, MA.

Eo, J. y B. Yil. Effects of ethylene, abscisic acid and auxin on fruit abscission in water dropwort (Oenanthe stolonifera DC.) Sci. Hortic. 123(2), 224-227. Doi: 10.1016/j.scienta.2009.08.011

Fallahi, E., D. Neilsen, G. Neilsen, B. Fallahi y B. Shafii. 2010. Efficient irrigation for optimum fruit quality and yield in apples. HortScience 45(11), 1616-1619.

Fischer, G., P.J. Almanza-Merchán y D. Miranda. 2014. Importancia y cultivo de la uchuva (Physalis peruviana L.). Rev. Bras. Frutic. 36(1), 1-15. Doi: 10.1590/0100-2945-441/13

Fischer, G., G. Ebert y P. Lüdders. 2007. Production, seeds and carbohydrate contents of cape gooseberry (Physalis peruviana L.) fruits grown at two contrasting Colombian altitudes. J. Appl. Bot. Food Qual. 81, 29-35.

Fischer, G., P. Lüdders y F. Torres. 1997. Influencia de la separación del cáliz de la uchuva (Physalis peruviana L.) sobre el desarrollo del fruto. Rev. Comalfi 24(1-2), 3-16.

Fischer, G. and O. Martínez. 1999. Calidad y madurez de la uchuva (Physalis peruviana L.) en relación con la coloración del fruto. Agron. Colomb. 16(1-3), 35-39.

Fischer, G. y J.O. Orduz-Rodríguez. 2012. Ecofisiología en frutales. pp. 54-72. En: Fischer, G. (ed.). Manual para el cultivo de frutales en el trópico. Produmedios, Bogotá.

Fischer, G., C. Ulrichs y G. Ebert. 2015. Contents of nonstructural carbohydrates in fruiting cape gooseberry
(Physalis peruviana L.) plants. Agron. Colomb. 33(2), 155-163. Doi: 10.15446/agron.colomb.v33n2.51546

Gordillo, O., G. Fischer y R. Guerrero. 2004. Efecto del riego y de la fertilización sobre la incidencia del rajado en frutos de uchuva (Physalis peruviana L.) en la zona de Silvania (Cundinamarca). Agron. Colomb. 22(1), 53-62.

Guerrero, B., M. Velandia, G. Fischer y H. Montenegro. 2007. Los ácidos carboxílicos de extractos vegetales y la humedad del suelo influyen en la producción y el rajado del fruto de uchuva (Physalis peruviana L.). Rev. Colomb. Cienc. Hortic. 1(1), 9-19. Doi: 10.17584/rcch.2007v1i1.1141

Icontec. 1999. Frutas frescas. Uchuva. Especificaciones. Norma Técnica Colombiana NTC 4580. Instituto Colombiano de Normas Técnicas, Bogotá.

Léchaudel, M. y J. Joas. 2007. An overview of preharvest factors influencing mango fruit growth quality and postharvest behaviour. Braz. J. Plant Physiol. 19, 287-298. Doi: 10.1590/S167704202007000400004

Martínez, F., J. Sarmiento, G. Fischer y F. Jiménez. 2008. Efecto de la deficiencia de N, P, K, Ca, Mg y $\mathrm{B}$ en componentes de producción y calidad de la uchuva (Physalis peruviana L.). Agron. Colomb. 26(3), 389-398

Oliveira, V.H., F.R. Miranda, R.N. Lima y R.R. Cavalcante. 2006. Effect of irrigation frequency on cashew nut yield in northeast Brazil. Sci. Hortic. 108, 403-407. Doi: 10.1016/j.scienta.2006.02.003

Porro, D., M. Ramponi, T. Tomasi, L. Rolle y S. Poni. 2010. Nutritional implications of water stress in grapevine and modifications of mechanical properties of berries. Acta Hortic. 868, 73-80. Doi: 10.17660/ActaHortic.2010.868.5

Ramachandra-Reddy, A., K. Chaitanya y M. Vivekanandan. 2004. Drought induced responses of photosynthesis and antioxidant metabolism in higher plants. J. Plant Physiol. 161, 1189-1202. Doi: 10.1016/j.jplph.2004.01.013

Ramírez, F., G. Fischer, T.L. Davenport, J.C.A. Pinzón y C. Ulrichs. 2013. Cape gooseberry (Physalis peruviana L.) phenology according to the $\mathrm{BBCH}$ phenological scale. Sci. Hortic. 162, 39-42. Doi: 10.1016/j. scienta.2013.07.033

Román, L. y M. Gutiérrez. 1998. Evaluación de ácidos carboxílicos y nitrato de calcio para incrementar calidad, cantidad y vida de anaquel en tres tipos de melón. Terra Latinoamericana 16(1), 49-54. 
Rufat, J., A. Arbonés, P. Villar, X. Domingo, M. Pascual y J.M. Villar. 2010. Effects of irrigation and nitrogen fertilization on growth, yield and fruit quality parameters of peaches for processing. Acta Hortic. 868, 87-94. Doi: 10.17660/ActaHortic.2010.868.7

Silber, A. y A. Bar-Tal. 2008. Nutrition of substratesgrown plants. pp. 291-339. En: Raviv, M. y J.H. Lieth (eds.). Soilless culture: Theory and practice. Elsevier, Amsterdam, The Netherlands.

Silber, A., G. Xu, I. Levkovitch, S. Soriano, A. Bilu y R. Wallach. 2003. High fertigation frequency: the effects on uptake of nutrients, water, and plant growth. Plant Soil 253(2), 467-477. Doi: 10.1023/A:1024857814743

Spreer, W., M. Nagle, S. Neidhart, R. Carle, S. Ongprasert y J. Muller. 2007. Effect of regulated deficit irrigation and partial rootzone drying on the quality of mango fruits (Mangifera indica L. cv. 'Chok Anan'). Agr. Water Manage. 88, 173-180. Doi: 10.1016/j.agwat.2006.10.012

Taiz, L. y E. Zeiger. 2010. Plant physiology. $5^{\text {th }}$ ed Sinauer Associates, Sunderland, MA.

Treeby, M., R. Henriod, K. Bevington, D. Milne and R. Storey. 2007. Irrigation management and root- stock effects on navel orange (Citrus sinensis (L.) Osbeck) quality. Agr. Water Manage. 91, 24-32. Doi: 10.1016/j.agwat.2007.04.002

Veit-Köhler, U., A. Krumbein y H. Kosegarten. 1999. Effect of different water supply on plant growth and fruit quality of Lycopersicon esculentum. J. Plant Nutr. Soil Sci. 162(6), 583-588. Doi: 10.1002/ (SICI)1522-2624(199912)162:6<583::AID JPLN583>3.0.CO;2-P

Vélez, J.E. y J.G Álvarez. 2012. Riego en frutales. pp. 186-204. En: Fischer G. (ed.), Manual para el cultivo de frutales en el trópico. Produmedios, Bogotá.

Villamizar, F., A. Ramírez y M. Menes. 1993. Estudio de la caracterización física, morfológica y fisiológica de la uchuva (Physalis peruviana L.). Agro-Desarrollo 4(1-2), 305-320.

Wang, F.X., Y. Kangy S.P. Liu. 2006. Effects of drip irrigation frequency on soil wetting pattern and potato growth in North China Plain. Agr. Water Manage. 79(3), 248-264. Doi: 10.1016/j.agwat.2005.02.016

Zegbe-Domiguze, J.A., M.H. Behboudian y B.E. Clothier. 2004. Partial root zone drying is a feasible option for irrigating processing tomatoes. Agr. Water Manage. 68, 195-206. Doi: 10.1016/j.agwat.2004.04.002 